\title{
"Abgassimulator zur Nachbildung und Messung von Abgasen aus unterschiedlichen industriellen Prozessen zur Entwicklung und Untersuchung von Sensoren- und Messsystemen unter bekannten und reproduzierbaren Betriebsbedingungen"
}

\author{
Dr. -Ing. Mohammadshayesh Aleysa ${ }^{1}$, Dr. Judit Angster ${ }^{2}$, Dr. Andras Miklós ${ }^{2}$, M.Sc. Niro Akbary ${ }^{1}$, \\ M.Sc. Martin Walter ${ }^{1}$ \\ ${ }^{1}$ Fraunhofer-Institut für Bauphysik, Fachgebiet Verbrennungssysteme, Nobelstraße 12, 70569 \\ Stuttgart (Deutschland) \\ ${ }^{2}$ Fraunhofer-Institut für Bauphysik, Fachgebiet Photoakustik, Nobelstraße 12, 70569 Stuttgart
}

(Deutschland)

\section{Zusammenfassung}

Der Abgassimulator des Fraunhofer IBP wurde für die Nachbildung und Messung von Abgasen aus unterschiedlichen industriellen Prozessen im Rahmen eines von der Deutschen Bundesstiftung Umwelt (DBU) geförderten Projekts entwickelt. Diese innovative Einrichtung ist so konzipiert und flexibel konstruiert, dass vielfältige Prozessuntersuchungen unter bekannten und reproduzierbaren Bedingungen im Dauerbetrieb durchführbar sind. Der Abgassimulator verfügt über hochgenaue Messeinrichtungen zur Erfassung von staub- und gasförmigen Komponenten sowie zur Ermittlung der nötigen Abgasparameter (Abgasmassenstrom, Abgastemperatur, Abgasdruck, Feuchtegehalt). Dank der innovativen Bauweise wurde der Abgassimulator für mehrere Anwendungen wie z. B. zur Entwicklung photoakustischer Feinstaub-Sensorik, zur Untersuchung des Verhaltens der Lambdasonde bei unterschiedlichen Konzentrationen unverbrannter Bestandteile im Abgas und zur Untersuchung der Abscheideleistung von Staubabscheidern und Katalysatoren eingesetzt. In diesem Beitrag wird die Anwendung des Abgassimulators zur Entwicklung eines photoakustischen Feinstaubmesssensors sowie zur Untersuchung der Oxidationsleistung von staub- und gasförmigen Emissionen in unterschiedlichen Strukturen dargestellt.

Keywords: Abgassimulator, Rußpartikelgenerator, Rußpartikelmessung, photoakustische Spektroskopie, Filter-Reaktor-System

\section{Abgassimulator des Fraunhofer IBP}

Der Abgassimulator des Fraunhofer IBP, dargestellt in Abb. 1, wurde so konzipiert und konstruiert, dass sowohl Abgas als auch Abluft aus unterschiedlichen industriellen Prozessen im realen sowie heißen Zustand nachgebildet und die dazugehörigen Prozesse entsprechend simuliert werden können. Hierbei wurde großer Wert darauf gelegt, dass der Abgassimulator vielfältig und für den Dauerbetrieb eingesetzt werden kann. Im Folgenden werden die Charakteristika und technischen Besonderheiten des entwickelten Abgassimulators dargestellt:

- Der Abgassimulator zeichnet sich durch eine hohe technische Modifizierbarkeit aus. Je nach Anwendungsfall ist dieser dank der innovativen Bauweise sowie des verwendeten Abgasrohrsystems flexibel umkonstruier- und erweiterbar, sodass ein vielfältiger Einsatz mit vertretbarem Aufwand gewährleistet werden kann. Dabei können unterschiedliche Durchmesser des Abgasrohrsystems realisiert sowie unterschiedliche Abluft- und Abgasmengen durch die verwendete modulierbare Porenbrennertechnik erzeugt und simuliert werden.

- Beim Betrieb des Abgassimulators sind die physikalischen, chemischen sowie thermodynamischen Parameter des zu untersuchenden Mediums in großen Bereichen und vielfältig einstellbar. Die Einstellung dieser Parameter erfolgt unter bekannten und reproduzierbaren Betriebs- und Rahmenbedingungen. Dadurch können komplizierte Messungen mit hoher Wiederholbarkeit, Reproduzierbarkeit und Genauigkeit durchgeführt werden. 
- Die technische Einrichtung ermöglicht die Simulation von Abgas und Abluft aus unterschiedlichen industriellen Prozessen. Es lassen sich komplexe und ungünstige Betriebsfälle (WorstCase-Szenarien) im Dauerbetrieb untersuchen, die im realen Betrieb nur unter bestimmten Umständen und mit entsprechenden Risiken sowie hohem Aufwand eingestellt werden können.

- Der Abgassimulator verfügt über hochgenaue und zuverlässige Messeinrichtungen zur Erfassung von staub- und gasförmigen Komponenten sowie zur Messung der nötigen Abgasparameter (Temperatur, Druck, Feuchtegehalt usw.), welche für die Auswertung und Umrechnungen nötig sind.

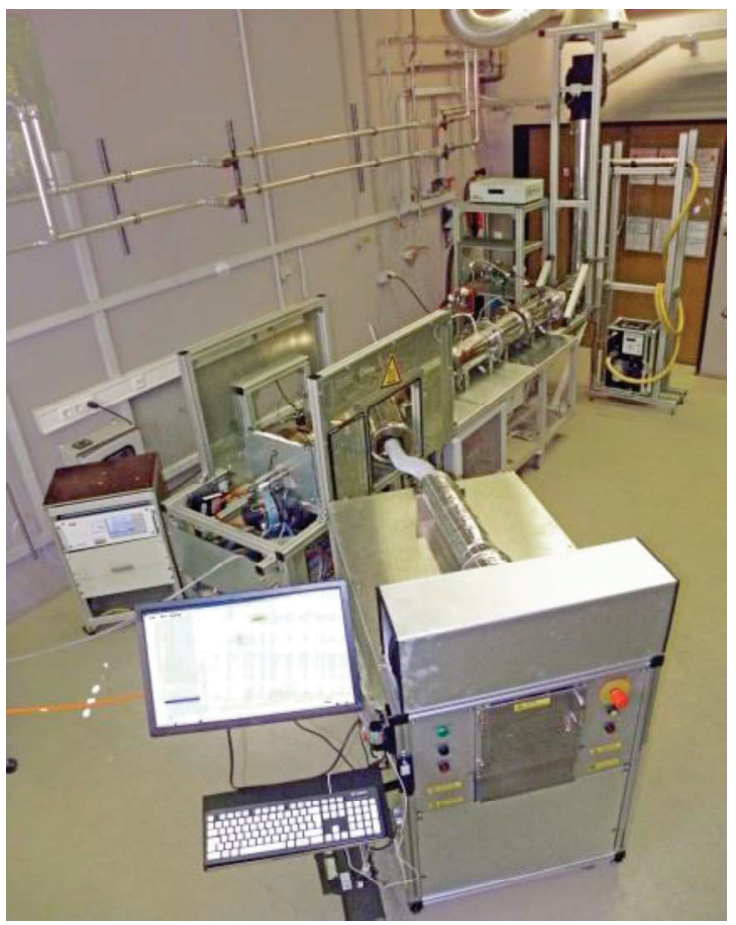

Abb.1: Technischer Aufbau des Abgassimulators des Fraunhofer IBP.

\section{Technischer Aufbau des Abgaslabor-systems}

Im Wesentlichen besteht der Abgassimulator aus:

\section{Einrichtung zur Erzeugung des Grund- Abgases}

Zur Erzeugung des Grund-Abgases mit beliebigen Temperaturen und Volumenströmen wird die Porenbrennertechnik eingesetzt (Abb. 2). Diese Verbrennungstechnik gewährleistet sowohl eine schadstoffarme, als auch eine sehr stabile Verbrennung im Dauerbetrieb. Es werden gasförmige Brennstoffe eingesetzt.
Das Grund-Abgas setzt sich aus Kohlendioxid $\left(\mathrm{CO}_{2}\right)$, Wasser $\left(\mathrm{H}_{2} \mathrm{O}\right)$, Sauerstoff $\left(\mathrm{O}_{2}\right)$ und Stickstoff $\left(\mathrm{N}_{2}\right)$ zusammen.

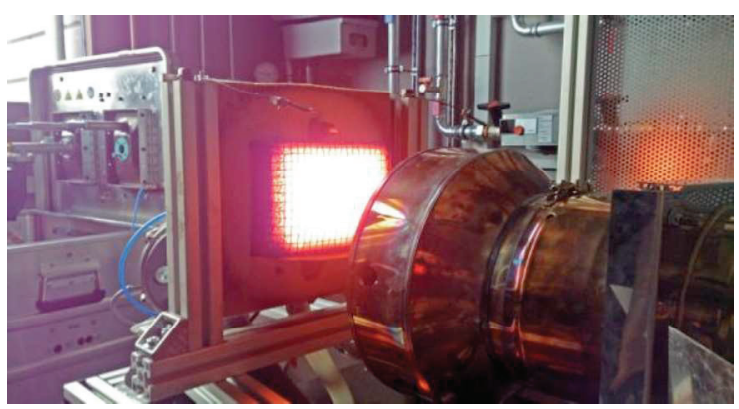

Abb.2: Porenbrenner zur Erzeugung des Grundabgases.

\section{Einrichtung zur Bereitstellung und Zugabe von staub- und gasförmigen Komponenten (Schadstoffe)}

Durch die kontrollierte Zugabe staub- und gasförmiger Komponenten werden definierbare Konzentrationen gewünschter Schadstoffemissionen erzeugt und Abluft- und Abgasprozesse entsprechend simuliert. Es wird zwischen der Zugabe von staub- und gasförmigen Schadstoffkomponenten unterschieden, welche entweder synthetisch bereitgestellt oder vor Ort erzeugt und in den Abgassimulator zugegeben werden.

\section{Bereitstellung und Zugabe gasförmiger Schadstoffemissionen}

Die gasförmigen Komponenten werden synthetisch bereitgestellt. Folgende Gase können in den Abgassimulator eingespeist werden:

- Brennbare gasförmige Komponenten wie z. B. Kohlenmonoxid (CO), Methan $\left(\mathrm{CH}_{4}\right)$ und Propan $\left(\mathrm{C}_{3} \mathrm{H}_{8}\right)$.

- Nicht-brennbare, gasförmige Komponenten wie z. B. Stickstoffmonoxid (NO), Stickstoffdioxid $\left(\mathrm{NO}_{2}\right)$, Schwefeldioxid $\left(\mathrm{SO}_{2}\right)$ Chlorwasserstoff $(\mathrm{HCl})$ und Fluorwasserstoff (HF).

- Sonstige Schadgase wie Schwefelwasserstoff $\left(\mathrm{H}_{2} \mathrm{~S}\right)$, Ammoniak $\left(\mathrm{NH}_{3}\right)$, Distickstoffmonoxid bzw. Lachgas $\left(\mathrm{N}_{2} \mathrm{O}\right)$.

Die Einspeisung der Gase in den Abgassimulator erfolgt durch eine eigenentwickelte Vorrichtung bzw. Methodik. Hierbei werden die Schadgase einer Mischstation über Druckminderer, Gasventile und Massendurchflussregler zugeführt, dort mit Druckluft vermischt und in den Abgassimulator transportiert, bevor sie anschließend in den Kernstrom des Abgases zugegeben werden. 
Um eine gleichmäßige Konzentrationsverteilung im gesamten Abgasstrom, unabhängig von der im Abgasrohr strömenden Abgasmenge zu erreichen, werden diese Schadgase mit hoher Geschwindigkeit mittels Druckluft über eine spezielle Lanze im Gegenstrom zugegeben (Abb. 3). Diese Methode der Zugabe hat sich in der Praxis sehr gut bewährt. Die Aufgabe von Massendurchflussreglern besteht darin, eine konstante Zugabe jeweiliger Schadgase sowie die Einstellung der Konzentration zu gewähr-

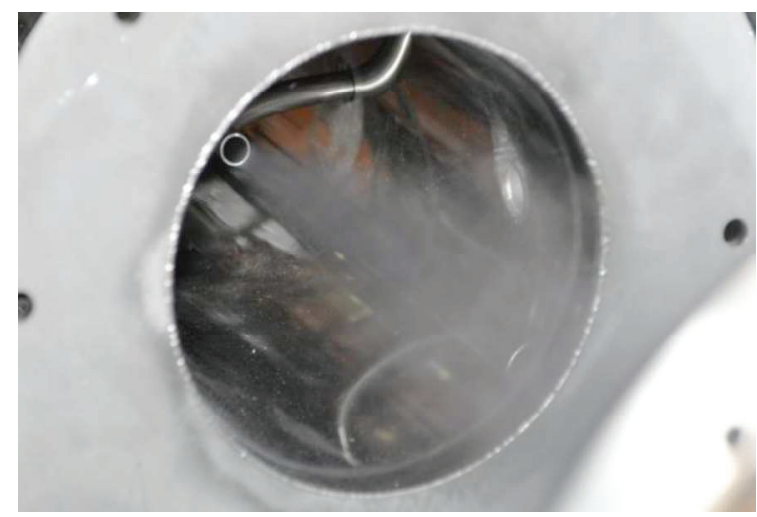

Abb.3: Spezielle Lanze für die Zugabe von staub- und gasförmigen Emissionen in den Abgassimulator.

leisten. Mit Hilfe einer SPS (speicherprogrammierbare Steuerung) werden die Massendurchflussregler gesteuert und somit die Zugabe grob geregelt. Die eingestellten Abgaskonzentrationen werden sekündlich gemessen und in einer Datenbank stetig abrufbar für die Auswertung gespeichert. Um eine repräsentative Probeentnahme bei der Messung der gasförmigen Komponenten, unabhängig von der im Abgasrohr strömenden Abgasmenge zu erhalten, wird eine spezielle Messstrecke verwendet. Diese Strecke besteht aus einem glattwandigen Rohrstück mit einer Verengung des Querschnitts (von $150 \mathrm{~mm}$ auf $80 \mathrm{~mm}$ ). Die Abgasprobe wird dem gesamten Querschnitt der Messstrecke über zwei Rohre mit einem Durchmesser von $8 \mathrm{~mm}$ entnommen. Diese Rohre sind zentrisch gekreuzt im Querschnitt der Messstrecke angeordnet und verfügen über 20 Probeentnahmeöffnungen (jeweils 10 Öffnungen), die über die gesamte Rohrlänge auf den beiden Strömungsseiten des Rohrs verteilt sind. Die entnommene Probe wird zentral durch ein Sammelrohr gesammelt, der Abgasanalyse zugeführt und dort analysiert.

\section{Bereitstellung und Zugabe staubförmiger Komponenten}

Bei den staubförmigen Schadstoffemissionen ist zwischen groben (im Mikrometerbereich) und feinen Stäuben (im Nanometerbereich) zu unterscheiden. Grobe Stäube mit industriellem Ursprung werden synthetisch mit definierten Partikelgrößen und bestimmten mittleren Korngrößenverteilungen bereitgestellt. Hier sind sowohl organische, als auch anorganische Stäube einsetzbar. Zur Dosierung dieser Stäube in den Abgassimulator wird das Dosierund Dispergiersystem (RBG 1000I) der Firma PALAS eingesetzt (Abb. 4). Dieses System (RBG 1000l) kann sowohl organische (Ruß), als auch anorganische Stäube (Salze) mit einem Durchsatz von 0,04 bis $430 \mathrm{~g} / \mathrm{h}$ (bei angenommener Stopfdichte von $1 \mathrm{~g} / \mathrm{cm}^{3}$ )

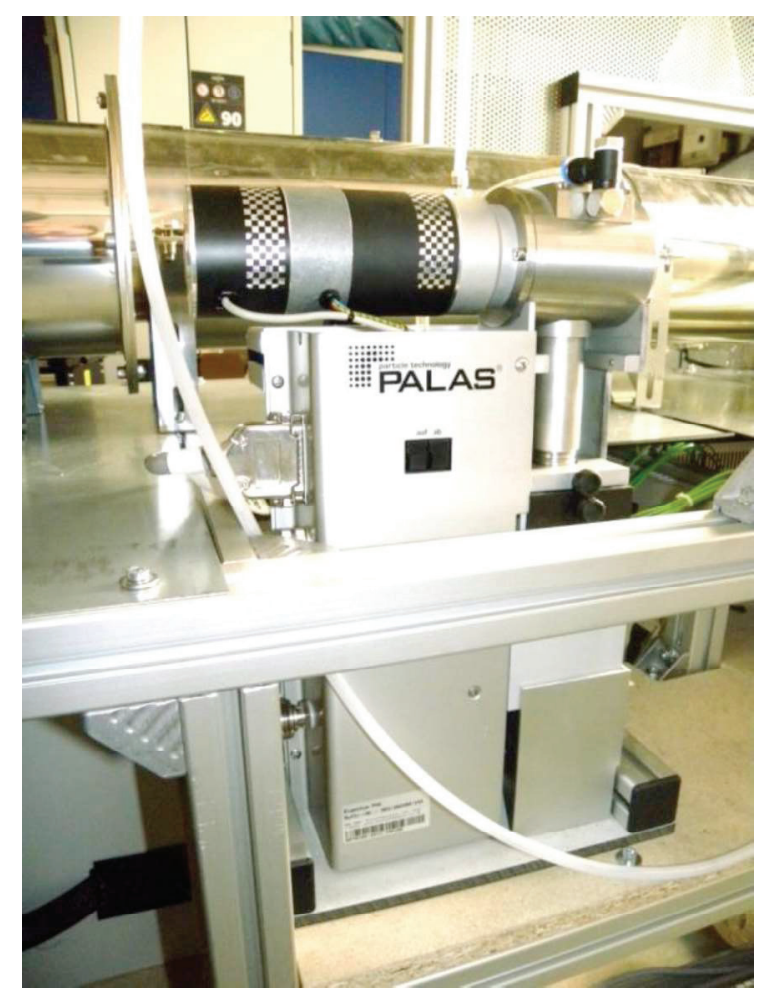

Abb.4: Dosier- und Dispergiersystem RBG 10001.

und Partikelgrößen zwischen 0,1 $\mu \mathrm{m}$ bis 100 $\mu \mathrm{m}$ dosieren. Der besondere Vorteil dieses Systems liegt darin, dass es für den Dauerbetrieb sowie für die Dosierung und Dispergierung komplexer Stäube wie z. B. fein gemahlene Verbrennungsasche geeignet ist. Die zu dosierenden Stäube werden in den zylindrischen Feststoffbehälter eingefüllt und mit einem Stopfer verdichtet. Nach der Einfüllung wird der Behälter in den Dispergierkopf des RGB eingebaut. Die verdichteten Stäube werden mit einem geregelten Transportkolben gegen eine rotierende Bürste vorgeschoben und dort mit Hilfe eines einstellbaren Druckluftstroms in den Abgassimulator und die Lanze transportiert. Um eine konstante gleichmäßige Zugabe und 
reproduzierbare Messungen zu erreichen, sind Anforderungen an das Dosiersystem, an die verwendeten Staubpartikel sowie an die Strömung im Abgasrohr einzuhalten. Hier sollten folgende Punkte besonders berücksichtigt werden:

- Die verwendeten Staubpartikel sollten keine kohäsiven Eigenschaften aufweisen. Kohäsive Stäube lassen sich nur ungleichmäßig dosieren.

- Art der Einfüllung des Staubbehälters des Dosier- und Dispergiersystems. Die Verdichtung der Stäube spielt eine besondere Rolle.

- Die Abgasgeschwindigkeiten im Abgasrohr sollen deutlich höher, als die Sedimentationsgeschwindigkeiten der Staubpartikel, eingestellt werden. Die Dichte der Staubpartikel sollte geringer, als die Dichte des produzierten Abgases sein.

- Vor der Probenentnahme dürfen die Staubpartikel nicht elektrisch aufgeladen werden, da sie ansonsten an der Innenwand des Abgasrohrs abgeschieden werden.

- Sehr feine Stäube $(<\mu \mathrm{m})$ können zwar dosiert werden, aber die Einstellung gewünschter Konzentrationen ist nur schwierig zu erreichen.

Organische Feinstäube (von ca. $10 \mathrm{~nm}$ bis $>200 \mathrm{~nm}$ ) werden durch einen RußpartikelGenerator der Firma PALAS (defined Soot Particle Generator DSP 3000 H) produziert und im warmen Zustand bei einer Temperatur von $250{ }^{\circ} \mathrm{C}$ in den Abgassimulator eingespeist (Abb. 5).

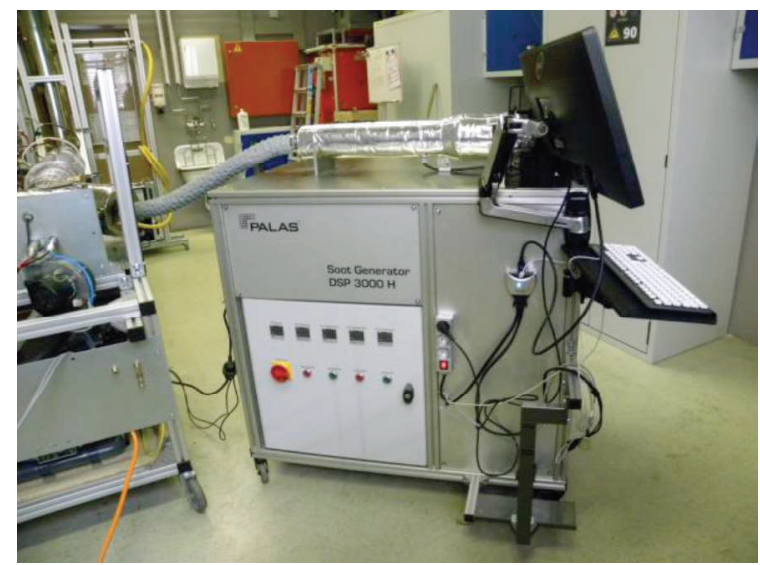

Abb.5: Ruß-Generator DSP 3000 H der Firma PALAS.

Durch die Einstellung definierter Verbrennungszustände des Gasbrenners anhand der Einstellung des Brennstoff-Luft-
Mischungsverhältnisses (Lambda), kann eine bestimmte mittlere Partikelgröße und ein Partikelmassenstrom (von ca. $50 \mathrm{mg} / \mathrm{h}$ bis ca. $3 \mathrm{~g} / \mathrm{h}$ ) erzeugt werden. Hier gilt, je unvollständiger die Verbrennung (Lambda < 1) ist, desto höher sind die zu erreichenden Rußpartikelkonzentrationen bzw. desto gröber sind die produzierten Partikel. Die genaue Einstellung der Partikelkonzentrationen (Partikel/ $\mathrm{cm}^{3}$ ) sowie der Korngrößenverteilung $(5 \mathrm{~nm}$ bis $350 \mathrm{~nm})$ erfolgt durch eine reale Messung mit dem SMPS (Scanning Mobility Particle Sizer). Die Abgasprobe wird nach Erreichen eines konstanten Betriebszustandes (konstante Temperatur, konstanter Abgasvolumenstrom und stabiler Druck) entnommen und analysiert. Die Besonderheit des im DSP $3000 \mathrm{H}$ verwendeten Brenners besteht in der Gewährleistung einer stabilen Partikelbildung bezüglich der Partikelgröße und Partikelkonzentration über eine lange Betriebszeit. Hier haben die Umgebungsbedingungen, wie Temperatur und Umgebungsdruck, nur sehr geringen Einfluss auf die Rußbildung. Mit Hilfe einer Software kann der gesamte Rußpartikel-Generator gesteuert und die gewünschten Betriebszustände zur Erzeugung vordefinierter Partikelgrößen und Partikelkonzentrationen eingestellt werden. Auf einem Display wird das Fließschema des DSP 3000 mit den jeweiligen Online-Werten aufgezeigt.

\section{Messeinrichtung}

Der Abgassimulator ist mit hochgenauen Messeinrichtungen zur Messung von staubund gasförmigen Emissionen sowie zur Messung von Abgasparametern (wie z. B. Temperatur, Druck, Abgasgeschwindigkeit, Abgasvolumenstrom usw.) ausgerüstet, die für die Einstellung gewünschter Betriebszustände und für die Umrechnung der gemessenen Emissionswerte benötigt werden. Zur Abgasanalyse werden sowohl heißextraktive (GASMET: TTGASSYS001der Firma Ansyco), als auch kaltextraktive (Advance Optima AO 2000 der Firma ABB AUTOMATION) Abgasanalysegeräte verwendet. Sie haben den Vorteil, dass sie für den Einsatz bei rauen Betriebsbedingungen sowie für den Dauerbetrieb geeignet sind. Folgende Abgaskomponenten lassen sich kontinuierlich und mit hoher Genauigkeit messen:

- Typische Verbrennungsprodukte: Kohlendioxid $\left(\mathrm{CO}_{2}\right)$, Wasserdampf $\left(\mathrm{H}_{2} \mathrm{O}\right)$ und Sauerstoff $\left(\mathrm{O}_{2}\right)$.

- Brennbare Abgaskomponenten aus unvollständiger Verbrennung: Kohlenmonoxid (CO), Gesamtkohlenwasserstoffe $\left(\mathrm{C}_{n} \mathrm{H}_{m}\right)$. 
- Nicht-brennbare saure Abgaskomponenten: Stickstoffmonoxid

(NO), Stickstoffdioxid $\left(\mathrm{NO}_{2}\right)$, Schwefeldioxid $\quad\left(\mathrm{SO}_{2}\right), \quad$ Chlorwasserstoff $(\mathrm{HCl})$ und Fluorwasserstoff (HF).

Zur Messung der Gesamtkohlenwasserstoffe $\left(\mathrm{C}_{n} \mathrm{H}_{m}\right)$ wird auch der KohlenwasserstoffAnalysator Thermo-FID der Firma SKElektronik eingesetzt. Je nach verwendetem Kalibriergas werden die gemessenen Konzentrationen entweder als Methan $\left(\mathrm{CH}_{4}\right)$ oder als Propan $\left(\mathrm{C}_{3} \mathrm{H}_{8}\right)$ angegeben. Außerdem können sonstige Abgase wie z. B. Ammoniak $\left(\mathrm{NH}_{3}\right)$, Distickstoffmonoxid $\left(\mathrm{N}_{2} \mathrm{O}\right)$, Ethen $\left(\mathrm{C}_{2} \mathrm{H}_{4}\right)$, Ethan $\left(\mathrm{C}_{2} \mathrm{H}_{6}\right)$, Pentan $\left(\mathrm{C}_{5} \mathrm{H}_{12}\right)$, Methanol $\left(\mathrm{CH}_{4} \mathrm{O}\right)$ oder Formaldehyd $\left(\mathrm{CH}_{2} \mathrm{O}\right)$ mit dem Abgasanalysator der Firma Ansyco (FTIR-Spektroskopie) nach einer entsprechenden Kalibrierung erfasst werden. Das System GRAVIMAT SHC502 der Firma Sick wird zur gravimetrischen Bestimmung von Staubemissionen verwendet. Dieses Staubmesssystem hat gegenüber herkömmlichen Staubmesssystemen den Vorteil, dass der Planfilter in einem speziellen Filterkopf direkt bei der Messung eingebaut und in die Abgasanlage eingeführt wird. Dieser Planfilter wird mit dem Filterkopf zusammen vor und nach der Probenentnahme abgewogen und die abgeschiedene Staubmenge entsprechend bestimmt.

Staubverluste durch lange Abgaswege, wie bei herkömmlichen Staubmesssystemen, entfallen. Folglich kann eine sehr genaue Staubmessung sowohl bei niedrigen als auch hohen Staubkonzentrationen realisiert werden. Zur Erfassung der Feinstäube wird das SMPSSpektrometer eigesetzt. Bei dem SMPS handelt es sich um einen Kondensationspartikelzähler (CPC: Condensation Particle Counter), der mit einem differentiellen Mobilitätsanalysator (DMA: Differential Mobility Analyser) zur Ermittlung der Korngrößenverteilung gekoppelt ist. Das SMPS ermöglicht die Messung der Feinstaubkonzentrationen (Partikelgröße von $5 \mathrm{~nm}$ bis $1 \mu \mathrm{m}$ ) sowie die Bestimmung der Korngrößenverteilung für einen Partikelgrößenbereich von $5 \mathrm{~nm}$ bis $350 \mathrm{~nm}$.

\section{Abgasfördersystem}

Das Abgasfördersystem besteht im Wesentlichen aus einem Saugzuggebläse, das bezüglich des gewünschten Unterdrucks im Abgassimulator zu steuern und $z u$ regeln ist. Das Abgasfördersystem hat die Aufgabe, die produzierte Abgasmenge während des Betriebs sicher aus dem Abgassimulator zu befördern. Das Abgas wird über eine Abgasabzugshaube in einen Schornstein geleitet, der ständig über hohen Unterdruck für die sichere Absaugung der produzierten Abgase sowie der Abluft im Raum sorgt. Hier soll dadurch eine Luftwechselzahl von 10/Stunde sowohl während als auch außerhalb des Betriebes eingestellt werden.

\section{Steuerungs- und Regeleinheit und Datenerfassungssystem}

Die technischen Bestandteile (Porenbrenner, Zugabe von staub- und gasförmigen Komponenten, Abgasfördersystem) des Abgassimulators werden durch eine selbstentwickelte Einheit gesteuert und geregelt. Bei dieser Steuerungs- und Regeleinheit handelt es sich um eine speicherprogrammierbare Steuerung (SPS). Zusätzlich zur Steuerung und Regelung wird die SPS zur hochqualitativen Datenerfassung und -speicherung verwendet. Die Abtastrate (Anzahl der Messungen je Sekunde oder kurz Abtastfrequenz) kann hier beliebig auch im Millisekundenbereich eingestellt werden. Außerdem können die Messwerte auf dem Display visualisiert und der Verlauf der Messung ständig kontrolliert werden. Der Vorteil der SPS bezüglich der Anwendung im Abgassimulator liegt darin, dass sowohl die Hardware als auch die Software, je nach Anwendungsfall erweiterbar ist.

\section{Beispiele für mögliche Anwen- dungen des Abgassimulators}

Der Abgassimulator eignet sich für einen sehr breiten Anwendungsbereich. Nachfolgend werden Beispiele für mögliche Anwendungen dargestellt:

- Entwicklung und Bewertung von Abgasbehandlungssystemen unter reproduzierbaren Bedingungen wie z. B.:

$$
\begin{aligned}
& \text { - Ermittlung von undigen } \\
& \text { Betriebspunkten und -parametern, } \\
& \text { Abscheideleistung von staub- und } \\
& \text { gasförmigen Schadstoffemissionen. } \\
& \text { - Aerodynamisches Verhalten bzw. } \\
& \text { Druckverlust. } \\
& \text { - Einfluss von Abgasparametern und } \\
& \text { Abgaskomponenten auf die Funktion, } \\
& \text { Effektivität und Beständigkeit } \\
& \text { katalytischer Beschichtungen. } \\
& \text { - Aufnahmekapazität von Ab- und } \\
& \text { Adsorptionsmitteln. }
\end{aligned}
$$

- „Stresstest“ für technische Abgaskomponenten (wie z. B. Sensoren, Abgasklappen, etc.). 
- Untersuchung des Alterungsverhaltens unterschiedlicher Aggregate (Ermittlung der Durchbruchkurve von Katalysatoren und Adsorbern usw.).

- Entwicklung und Kalibrierung von Abgasmesssystemen und -strecken.

Im Folgenden werden zwei Anwendungen für den Abgassimulator ausführlich beschrieben:

\section{Anwendungsbeispiele des Abgassimulators}

\section{Entwicklung eines photoakustischen Feinstaubmesssensors}

Im Rahmen der Forschungsaktivitäten des Fachgebiets Verbrennungssysteme sowie der Arbeitsgruppe Photoakustik des Fraunhofer IBP wurde eine photoakustische DurchlaufTestzelle zur selektiven Messung organischer Stäube bei der Verbrennung von Biomasse entworfen und gebaut. Die photoakustische Spektroskopie (PAS) ist eine Messmethode mit einer großen Empfindlichkeit, bei gleichzeitig sehr guter Selektivität. Dies sind zwei äußerst wichtige Voraussetzungen bei der Gasanalyse. Die photoakustische Wirkung basiert auf der sensiblen Erkennung von akustischen Wellen, die durch die Absorption von gepulstem oder moduliertem monochromatischem Licht erzeugt werden (Abb. 6).

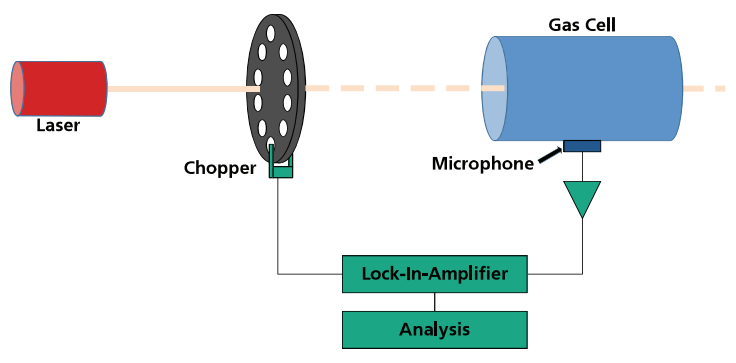

Abb.6: Schemadarstellung der photoakustischen Spektroskopie.

Prinzipiell können mit der PAS alle Gase gemessen werden, deren Konzentration auch durch optische Messungen (Absorptionsspektroskopie) bestimmt werden kann. Der wesentliche Vorteil der PAS ist jedoch, dass die Absorption direkt und nicht in Relation zum Hintergrund erfasst wird. Dadurch ist diese Messmethode äußerst genau und weist zudem eine sehr hohe Stabilität auf. Weitere Vorteile der PAS sind vor allem sehr schnelle Messungen (einige Sekunden). Zudem entfällt, im Gegensatz zu den bisher auf dem Markt verfügbaren Messgeräten zur Ermittlung von Staubemissionen, die nach jeder Messung erforderlichen aufwändigen Reinigungs- und Spülmaßnahmen der Sonde. Ebenfalls denk- bar ist der Einsatz der PAS im Bereich der Steuerung und Überwachung von Prozessen. Der Abgassimulator stellt aufgrund einer konstanten, gleichmäßigen Rußgeneration, unter wiederholbaren und reproduzierbaren Bedingungen eine geeignete Rußpartikelquelle zur Untersuchung bzw. gleichzeitigen Entwicklung und Optimierung einer neuen Zelle bzw. eines neuen Sensors zur Messung von organischen Staubemissionen in Abgasen dar. Darüber hinaus besteht die Möglichkeit variable Partikelmengen und Partikelgrößen, je nach eingestellter Luftüberschusszahl $\lambda$, zu produzieren. Die Einhaltung eines bestimmten $\lambda$-Werts hat großen Einfluss auf die Qualität der Verbrennung. Mit Hilfe dieser flexiblen Parametereinstellung ist die Simulation unterschiedlicher Qualitäten der Verbrennung realisierbar.

Im Verlauf von Vorversuchen wurde der Rußgenerator bei verschiedenen LuftzahlEinstellungen betrieben und die dabei produzierte Partikelkonzentration mit der PAS sowie parallel zur Ermittlung der Korngrößenverteilung mit dem SMPSSpektrometer gemessen.

In Abb. 7 werden die im Rahmen der Voruntersuchungen erhaltenen Ergebnisse aufgezeigt. Dargestellt ist die vom CPC gemessene Partikelmasse in $\mathrm{mg} / \mathrm{m}^{3}$ sowie die Rußpartikelkonzentration, ermittelt durch die PAS. Der Bereich des ausgegebenen Voltsignals wurde an die y-Achsenskalierung des CPC angepasst. Die Abbildung zeigt die parallel erfassten Rußpartikelkonzentrationen bei insgesamt sechs simulierten unterschiedlichen Lambda-Werten im Bereich zwischen $\lambda=0,45$ bis 0,70 .

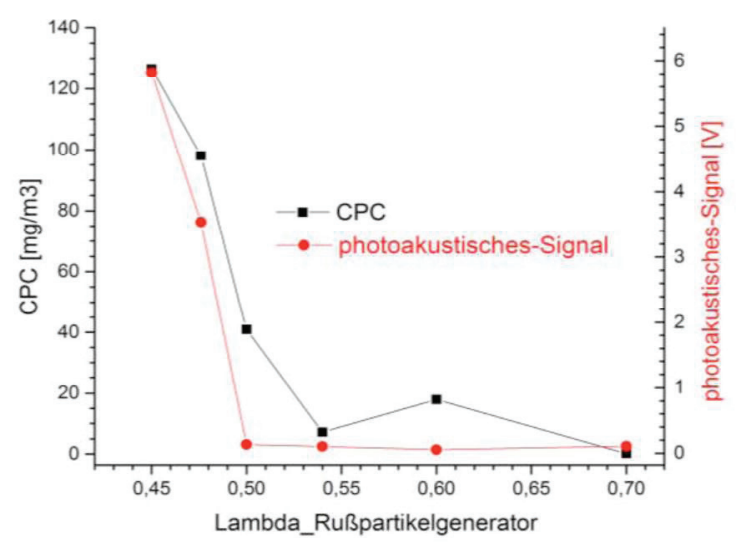

Abb.7: Bestimmung der Rußpartikelkonzentration bei definierten Lambda-Einstellungen durch einen Kondensations-partikelzähler sowie durch die PAS-Messmethode. 
Dem Verlauf aus Abb. 7 ist zu entnehmen, dass beide Messmethoden einen übereinstimmenden Trend bezüglich der erfassten Rußkonzentrationen in Abhängigkeit vom $\lambda$-Wert haben. Mit zunehmendem $\lambda$-Wert sinkt sowohl das PAS-Spannungssignal, als auch die durch das CPC ermittelte Partikelkonzentration. Zukünftig sind weitere Entwicklungsarbeiten bezüglich der PAS-Zelle notwendig. Aufgrund dessen soll ein neuer Sensor entwickelt werden, der direkt im heißen Abgas einsetzbar ist.

\section{Experimentelle Untersuchung der Kor- relation zwischen der Architektur von dreidimensionalen Strukturen und der Filtrations- und Oxidationsleistung von staub- und gasförmigen Emissionen}

Im Rahmen eines von DBU geförderten und vom Fachgebiet Verbrennungssysteme des Fraunhofer IBP durchgeführten Projekts, wurden zwei Strukturgruppen (deterministisch und nicht-deterministisch) mit Hilfe des Abgassimulators für den Einsatz in biomassebetriebenen Kleinfeuerungsanlagen, als Filter-Reaktor-System zur Minderung der staub- und gasförmigen Schadstoffemissionen untersucht.
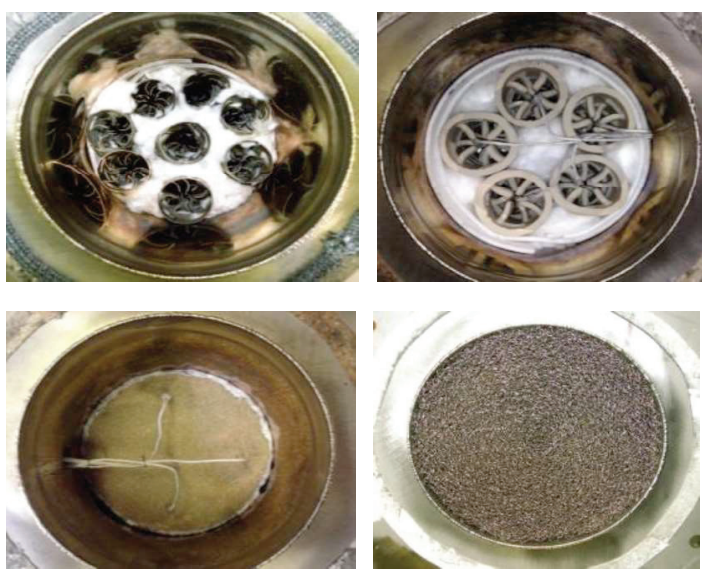

Abb.8: Aufbau der untersuchten Strukturen.

Dabei wurden keramische und metallische Füllkörper bzw. Pall-Ringe (als deterministische Struktur) sowie Drahtgestrick und Schaumkeramik (als nichtdeterministische Struktur) modulweise in den Abgassimulator gebaut und untersucht (vgl. Abb 8). Abb. 9 und Abb. 10 zeigen die Oxidationsleistung der untersuchten Strukturen. Es wird aus beiden Diagrammen ersichtlich, dass die Oxidationsleistung bei allen Strukturen mit der Erhöhung der Temperatur ansteigt und stark von der Verweilzeit im Modul abhängt. Bei metallischen und keramischen Füllkörpermodulen waren die Reduktionsgrade für Kohlenmonoxid und Propan deutlich höher als bei Schaumkeramik und Drahtgestrick. Diese effizientere Oxidationsleistung bzw. der höhere Reduktionsgrad für Kohlenmonoxid ist auf die längere Verweilzeit zurückzuführen.

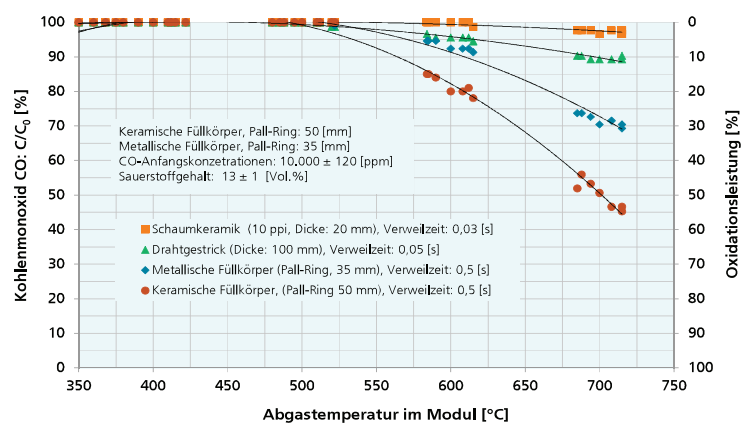

Abb 9: Reduktionsgrade von Kohlenmonoxid

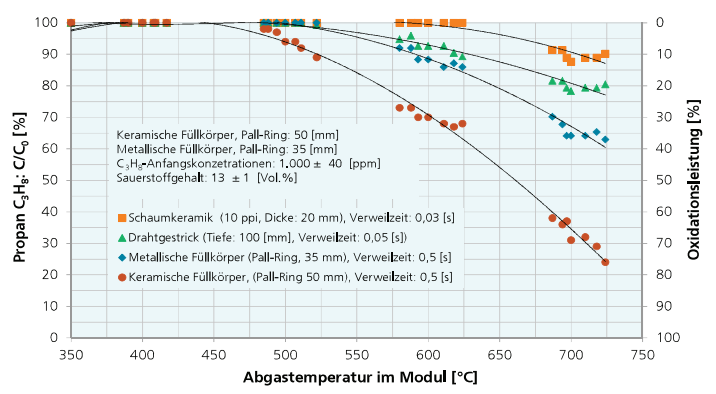

Abb 10: Reduktionsgrad des Propans.

Darüber hinaus ist anzumerken, dass die Oxidationsleistung beim Einsatz von keramischen Füllkörpern, bei gleicher Verweilzeit des Abgases, deutlich höher als beim Einsatz von metallischen Einbauten ist. Dieser Effekt kann auf unterschiedliche Ursachen zurückgeführt werden, die für die zielgerechte Entwicklung von Strukturen sehr interessant sein können. Hierbei ist sowohl die Architektur der Struktur, als auch das Strukturmaterial ausschlaggebend. Die Architektur der Struktur hat einen großen Einfluss auf die Art sowie die Intensität der Turbulenz und somit auf die lokale Sauerstoffverteilung innerhalb der Struktur. Das Material der Struktur ist durch seine spezifische Wärmekapazität, Wärmeleitfähigkeit und Wärmestrahlungsintensität im Wesentlichen für die Bereitstellung der gespeicherten Wärme und somit für die Oxidation verantwortlich.

\section{Literaturnachweis}

[1] M. Aleysa, M. Weclas, P. Leistner: "Korrelation der Filter-Reaktor-Architektur mit thermophysikalischen Funktionsbedingungen zur Erforschung und Entwicklung eines nichtkatalytischen 3D-Porösen Filter-ReaktorSystems für Biomassebetriebene Kleinfeuerungsanlagen."Abschlussbericht über ein Forschungsprojekt gefördert von der Deutschen Bundesstiftung Umwelt (AZ: 30550), S. 59 (2015) 